\title{
Four Classes of Intercellular Channels between Glial Cells in the CNS
}

\author{
Bruce M. Altevogt and David L. Paul \\ Program in Neuroscience and Department of Neurobiology, Harvard Medical School, Boston, Massachusetts 02115
}

Astrocytes form extensive gap junctions with other astrocytes and with oligodendrocytes. Junctional communication between CNS glia is likely of critical importance because loss of the gap junction channel-forming proteins, connexins $\mathrm{Cx} 32$ and $\mathrm{Cx} 47$, result in severe demyelination. However, CNS glia express at least six connexins, and the cellular origins and relationships of these proteins have not been determined. We produced a Cx29 reporter mouse in which the connexin coding sequence was replaced with a histological marker, which was used to demonstrate that $\mathrm{Cx} 29, \mathrm{Cx} 32$, and $\mathrm{Cx} 47$ are expressed specifically in oligodendrocytes. To determine the relationships between astrocyte and oligodendrocyte connexins, we used double- and triple-immunofluorescence microscopy using semithin sections $(<1 \mu \mathrm{m})$ of adult mouse spinal cord. Astrocytes form two distinct classes of gap junctions with each other; those composed of Cx26 and those composed of $\mathrm{Cx} 43$ and $\mathrm{Cx} 30$. In addition, astrocytes establish two classes of intercellular channels with oligodendrocytes, heterotypic $\mathrm{Cx} 26-\mathrm{Cx} 32$ channels and heterotypic $\mathrm{Cx} 30 / \mathrm{Cx} 43-\mathrm{Cx} 47$ channels that may also be heteromeric. In contrast, $\mathrm{Cx} 29 \mathrm{does}$ not colocalize with any of the other five connexins. The data provide the first in vivo demonstration of heterotypic intercellular channels and reveal an unexpected complexity in the composition of glial gap junctions.

Key words: oligodendrocyte; astrocyte; myelin; gap junction; connexin; intercellular communication

\section{Introduction}

All glial cell types and selected populations of neurons (Dermietzel and Spray, 1993) form gap junctions, which contain intercellular channels that directly connect the cytoplasm of adjoining cells. Intercellular channels are permeated by molecules up to $\sim 1$ $\mathrm{kDa}$ and thus provide a mechanism for chemical and electrical signaling between cells. In vertebrate organisms, intercellular channels are composed of connexins, a family of highly related proteins encoded by 20 or 21 genes (Evans and Martin, 2002). Intercellular channels are complex in structure compared with other ion channels because each cell independently contributes half of the channel (connexon). A connexon may contain a single (homomeric) or multiple (heteromeric) connexins. Similarly, the intercellular channel may be composed of identical (homotypic) or non-identical (heterotypic) connexons.

In virtually all regions of the CNS, gap junctions couple astrocytes into extensive networks (Rash et al., 2000). One possible function of this coupling is metabolic support, for example, the dispersion of $\mathrm{K}^{+}$taken up by the astrocyte after neuronal activity (Orkand et al., 1966). A second possible function involves intercellular signaling between astrocytes. An example of this is the propagation of $\mathrm{Ca}^{2+}$ waves between astrocytes, which appears to

Received July 11, 2003; revised Feb. 3, 2004; accepted March 9, 2004.

This work was supported by National Institutes of Health Grants R01 GM37751 (to D.L.P.), F31 NS41730 (to B.M.A.), R01 GM18974 (to Daniel A. Goodenough), and P30-HD18655 (to the Mental Retardation Research Center at Children's Hospital, Boston, MA). We are grateful for the expert technical assistance of Maria Ericsson, Rebecca Lewandowski, and Marta Mastroianni.

Correspondence should be addressed to David L. Paul, Department of Neurobiology, Harvard Medical School, 220 Longwood Avenue, Boston, MA 02115. E-mail: dpaul@hms.harvard.edu.

DOI:10.1523/JNEUROSCI.3303-03.2004

Copyright $\odot 2004$ Society for Neuroscience $\quad$ 0270-6474/04/244313-11\$15.00/0 be dependent on the expression of connexins (Goodenough and Paul, 2003). Because $\mathrm{Ca}^{2+}$-dependent glutamate release from astrocytes profoundly affects synaptic activity (Haydon, 2001), $\mathrm{Ca}^{2+}$ wave propagation could allow a network of astrocytes to influence synaptic activity over significant distances. Finally, it has been shown that astrocytes can sometimes establish intercellular channels directly with neurons, which could provide another mechanism for glial regulation of neuronal activity (Nedergaard, 1994; Alvarez-Maubecin et al., 2000).

Connexin expression in oligodendrocytes and Schwann cells is critical for normal myelination. Oligodendrocytes establish gap junctions with astrocytes, presumably recruiting oligodendrocytes into the astrocyte network, but junctions between oligodendendrocytes themselves are rarely if ever observed (Massa and Mugnaini, 1982; Waxman and Black, 1984; Rash et al., 2001). Animals lacking oligodendrocyte connexins exhibit severe deficits in CNS myelin, leading to early mortality (Menichella et al., 2003; Odermatt et al., 2003). Schwann cells also assemble gap junctions, although these junctions are found between the wraps of myelin within a single Schwann cell rather than between adjacent cells. These "reflexive" junctions likely act to shorten the path length for diffusion of small molecules between the periaxonal cytoplasm and the Schwann cell body (Balice-Gordon et al., 1998). Mutations in connexin32 (Cx32), expressed in both Schwann cells and oligodendrocytes, cause a common peripheral demyelinating neuropathy, X-linked Charcot-Marie-Tooth disease (Bergoffen et al., 1993).

Connexin expression in CNS glia is complex. Three connexins have been reported in astrocytes [Cx26 (Nagy et al., 2001), Cx30 (Kunzelmann et al., 1999), and Cx43 (Giaume et al., 1991)], although differences in their patterns of expression are observed. A 
different set of three connexins have been reported in oligodendrocytes [Cx29 (Altevogt et al., 2002), Cx32 (Dermietzel et al., 1989; Scherer et al., 1995), and Cx47 (Menichella et al., 2003)], and it has been suggested that Cx29 and Cx32 are expressed in a mutually exclusive subset of cells (Altevogt et al., 2002). It is not clear which connexins actually contribute to intercellular communication between oligodendrocytes and astrocytes, in part because the relative distributions of glial connexins are not well defined. To characterize their distributions, we used double and triple immunofluorescence on semithin sections $(<1 \mu \mathrm{m})$ of adult mouse spinal cord. In addition, we produced a Cx29 reporter mouse in which the connexin coding sequence was replaced with $\beta$-galactosidase $(\beta$-gal), a histological marker. We observed Cx29, Cx32, and Cx47 expression in all spinal cord oligodendrocytes but with different subcellular distributions. Oligodendrocyte Cx29 did not colocalize with any of the other five connexins. In contrast, oligodendrocyte Cx47 colocalized extensively with astrocyte $\mathrm{Cx} 30$ and $\mathrm{Cx} 43$, whereas oligodendrocyte $\mathrm{Cx} 32$ colocalized with astrocyte Cx26. Finally, astrocytes appeared to form two classes of gap junctions with each other, one containing Cx26 and the other containing $\mathrm{Cx} 43$ and $\mathrm{Cx} 30$. These data provide the first in vivo demonstration of heterotypic intercellular channels and reveal an unexpected complexity in the composition of glial gap junctions.

\section{Materials and Methods}

Gene targeting. Cx29 homologous arms were PCR amplified from $\mathrm{BALB} / \mathrm{c}$ genomic DNA, subcloned into pCRII (Invitrogen, San Diego, $\mathrm{CA}$ ) for sequencing, and then transferred into the pDTA targeting vector (gift from Frank Gertler, MIT, Cambridge, MA). The 5' amplicon consisted of 1536 bp directly upstream to the Cx29 start codon (see Fig. 1 A). The 3' amplicon started 800 bp downstream of the Cx29 stop codon and was $3893 \mathrm{bp}$. The Cx29 coding sequence was replaced in frame with a nuclear-localized $\beta$-galactosidase (gift from S. Dymecki, Harvard Medical School, Boston, MA). The targeting construct was linearized with XhoI and electroporated into J1 embryonic stem cells. G418-resultant clones were screened by PCR using a $5^{\prime}$ primer external to the targeting vector and a $3^{\prime}$ primer within the neo cassette. Two of 102 clones displayed correct recombination. After Southern blot confirmation of recombination and karyotyping, one clone was selected for blastocyst injection. Loss of protein was confirmed by Western blot as described previously (Altevogt et al., 2002).

Cx29 knock-out (KO) progeny were genotyped by PCR using primer pair A-B (see Fig. 1) to detect the wild-type (WT) allele (700 bp) and primer pair $\mathrm{C}-\mathrm{D}$ to detect the $\mathrm{KO}$ allele $(1100 \mathrm{bp})$. $\mathrm{PCR}$ was performed in a final volume of $20 \mu \mathrm{l}$. The reaction was denatured at $95^{\circ} \mathrm{C}$ for $5 \mathrm{~min}$, followed by 40 cycles $\left(94^{\circ} \mathrm{C}\right.$ for $15 \mathrm{sec}, 66^{\circ} \mathrm{C}$ for $15 \mathrm{sec}$, and $72^{\circ} \mathrm{C}$ for 80 $\mathrm{sec})$.

Primers. The primers used were as follows: A, ATCTGTGCTGTGCTATTTGGAGT; B, ACAGGTTGTGCTGCCAATAC; C, CCGACGGCACGCTGATTGAAG; and D, ATGCGGTCGCGTTCGGTTGC

Preparation of Cx29 antibody. A bacterial fusion protein containing glutathione S-transferase (GST) plus the C-terminal portion of Cx29 (amino acids 220-258) was produced using the vector pGEX-4T-3 as described previously (Altevogt et al., 2002). The fusion protein was used to immunize guinea pigs (Pocono Rabbit Farm, Canadensis, PA), and the resultant antisera were affinity purified using column-bound fusion protein as described previously (Gabriels and Paul, 1998). Purified antisera did not work on Western blots. However, the pattern of immune staining on sections of spinal cord was identical to that of the previously characterized rabbit antiCx29 antibody (Altevogt et al., 2002). In addition, no immunostaining was observed in spinal cord obtained from the $\mathrm{Cx} 29 \mathrm{KO}$ (data not shown).

$\beta$-gal histochemistry and immunocytochemistry. Adult Cx29 WT and heterozygote mice were deeply anesthetized and perfused with $10 \mathrm{ml}$ of PBS, followed by $20 \mathrm{ml}$ of $4 \%$ paraformaldehyde in PBS. Sciatic nerve, spinal cord, and brain were obtained, frozen, and sectioned at $10 \mu \mathrm{m}$ in a cryostat. $\beta$-gal histochemistry was performed by incubating sections at $37^{\circ} \mathrm{C}$ with $1 \mathrm{mg} / \mathrm{ml} 5$-bromo-4-chloro-3-indolyl- $\beta$-D-galactopyranoside, $100 \mathrm{ng} / \mathrm{ml}$ nitro-blue-tetrazolium in PBS with $2 \mathrm{mM} \mathrm{MgCl}_{2}$ and 2 mM EGTA for 1-3 hr. For immunocytochemistry, slides were washed with PBS, blocked with $5 \%$ goat serum and $0.1 \%$ Triton X-100 in PBS, and incubated overnight at $4^{\circ} \mathrm{C}$ with combinations of the following primary antibodies: mouse anti-Cx32 [7C6.C7, 1:2 dilution (Li et al., 1996)], rabbit anti- $\beta$-gal (1:2500 dilution; Chemicon, Temecula, CA), rabbit anti-Cx47 [1:1000 dilution (Menichella et al., 2003)], mouse antiCC1 [APC (Ab-7), 1:20 dilution; Oncogene Research Products, Boston, MA), and mouse anti-parvalbumin (P3088, 1:1000 dilution; Sigma, St. Louis, MO). The monoclonal mouse anti- $\beta$-gal Ab (clone 40-1a; 1:10 dilution) was developed by Joshua Sanes (Washington University, St. Louis, MO) and was obtained from the Developmental Studies Hybridoma Bank developed under the auspices of the National Institute of Child Health and Human Development and maintained by the University of Iowa (Department of Biological Sciences, Iowa City, IA). After incubating with primary antibodies, slides were washed and incubated with AlexaFluor 568 and 488 secondary antibodies (1:800 dilution; Molecular Probes, Eugene, OR). Slides were mounted with Gel Mount (Biomeda, Foster City, CA), visualized on a Nikon (Tokyo, Japan) E800 microscope, and photographed with a SPOT RT digital camera (Diagnostic Instruments, Sterling Heights, MI), followed by image manipulation with Adobe Photoshop 6.0 (Adobe Systems, San Jose, CA)

Semi-thin immunocytochemistry. Adult Cx29 WT, Cx29 KO, and Cx32 KO mice were deeply anesthetized and perfused with $10 \mathrm{ml}$ of PBS, followed by $20 \mathrm{ml}$ of $2 \%$ paraformaldehyde and $0.1 \%$ glutaraldehyde in PBS. Spinal cords were dissected and cryoprotected by incubation in $30 \%$ sucrose overnight at $4^{\circ} \mathrm{C}$, embedded in $2.3 \mathrm{M}$ sucrose in PBS, and sec- 
tioned at $0.5 \mu \mathrm{m}$ using a Leica (Nussloch, Germany) ultracryomicrotome. Slides were washed in PBS, blocked with 5\% donkey serum and $0.1 \%$ Triton X-100 in PBS, and incubated overnight at $4^{\circ} \mathrm{C}$ with combinations of the following primary antibodies: rabbit anti-Cx26 (catalog \#71-0500, 1:50 dilution; Zymed, San Francisco, CA), mouse anti-Cx26 (\#33-5800, 1:100 dilution; Zymed), rabbit anti-Cx29 [1:10 dilution (Altevogt et al., 2002)], guinea pig anti-Cx29 (1:2 dilution), mouse antiCx30 (catalog \#33-2500, 1:100 dilution; Zymed), mouse anti-Cx32 [7C6.C7, 1:2 dilution (Li et al., 1996)], goat anti-Cx32 (SC-7258, 1:200 dilution; Santa Cruz Biotechnology, Santa Cruz, CA), mouse anti-Cx43 (catalog \#13-8300, 1:100 dilution; Zymed), rabbit anti-Cx43 (catalog \#71-0700, 1:50 dilution; Zymed), and rabbit anti-Cx47 [1:1000 dilution (Menichella et al., 2003)]. Slides were washed with PBS and incubated with combinations of the following secondary antibodies at 1:800 dilution: donkey anti-mouse IgG Cy2 (catalog \#715-225-150; Jackson ImmunoResearch, West Grove, PA), donkey anti-rabbit Cy2 (catalog \#711225-152; Jackson ImmunoResearch), donkey anti-guinea pig Cy3 (catalog \#706-165-148; Jackson ImmunoResearch), donkey anti-goat Cy3 (catalog \#705-165-147; Jackson ImmunoResearch), donkey antirabbit Cy5 (catalog \#711-175-152; Jackson ImmunoResearch), and donkey anti-mouse IgG Cy5 (catalog \#715-065-150; Jackson ImmunoResearch). Slides were processed and photographed as described in the preceding paragraph. Toluidine Blue staining of adjacent sections and differential interference contrast optics were used to differentiate white and gray matter in the sections of spinal cord.

\section{Results}

All oligodendrocytes express $\mathrm{Cx} 29, \mathrm{Cx} 32$, and $\mathrm{Cx} 47$, each with a unique subcellular distribution

Previously, we reported that oligodendrocytes ensheathing small-diameter fibers strongly expressed Cx29 with a preferential localization to the juxtaparanode, whereas large-diameter fibers exhibited relatively little signal and none associated with a particular cellular domain (Altevogt et al., 2002). This could reflect a selective expression of Cx29 in subpopulations of oligodendrocytes, a selective distribution of the protein, or both. To explore these possibilities, we generated a Cx29 reporter mouse, in which the $\mathrm{Cx} 29$ coding region was replaced with a nuclear-localized $\beta$-gal (Fig. $1 A$ ). The absence of Cx29 gene product was confirmed by PCR and Western blot (Fig. $1 B, C$ ). Cx29 KO mice were viable and fertile, and the ratio of genotypes obtained was mendelian. Neither KOs nor heterozygotes displayed gross anatomic or motor deficits (data not shown).

Distribution of the $\beta$-gal reporter in heterozygote brain (Fig. $1 D$ ) and spinal cord (data not shown) showed a pattern of expression consistent with an oligodendrocyte origin. Reporter was observed in a large number of cells with small somata, particularly abundant in white matter tracts but also evident in gray matter. Reporter was also detected in the Purkinje cell layer of the cerebellum (Fig. $1 D$, boxed area), which contains Purkinje cells and Bergmann glia but not oligodendrocytes (Castejon et al., 2002). To identify the cells expressing reporter in the Purkinje cell layer, we double labeled sections with antibodies to $\beta$-gal and parvalbumin, a well characterized marker for Purkinje neurons (Celio and Heizmann, 1981). $\beta$-gal and parvalbumin did not colocalize (Fig. $1 E$ ), suggesting that $\mathrm{Cx} 29$ was expressed by Bergmann glia.

The percentage of oligodendrocytes expressing Cx29 was assessed by comparing reporter distribution to CC1 (Bhat et al., 1996), a well characterized oligodendrocyte cell body marker. In spinal cord (Fig. 2A), virtually every CC1-positive cell (green; cytoplasm) also contained $\beta$-gal (red; nuclear localized). Similar results were obtained in cortex, corpus callosum, hippocampus, and cerebellar white matter (data not shown). Together, these data suggest that Cx29 is expressed by most if not all oligoden-
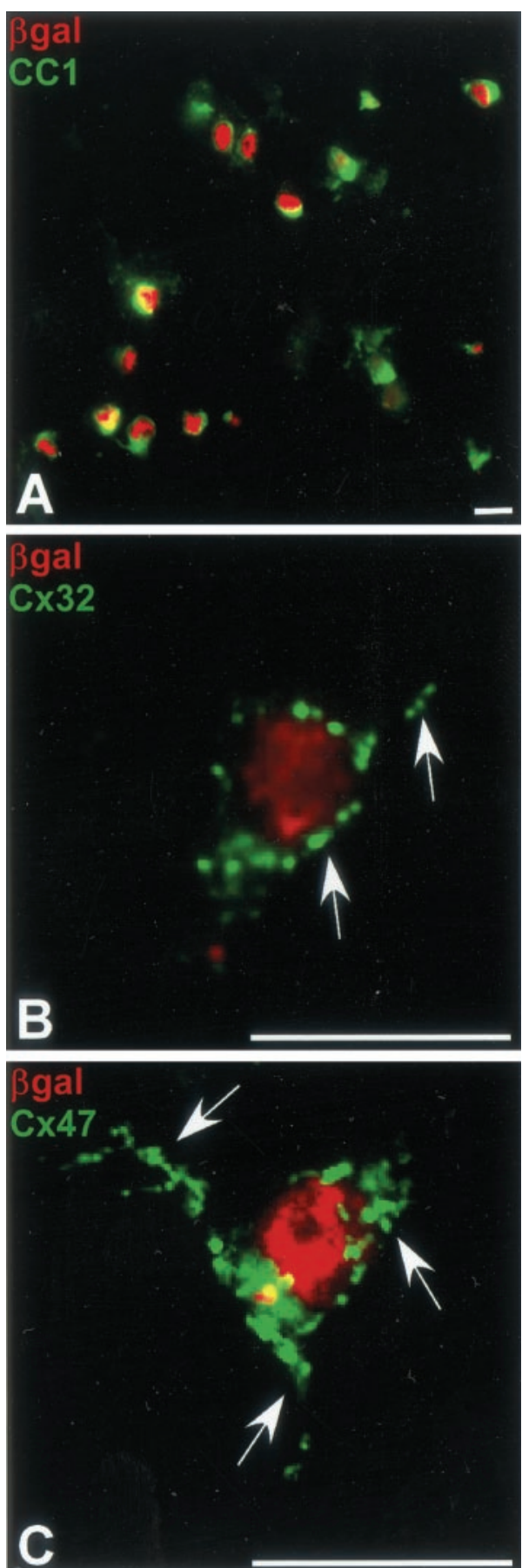

Figure 2. The majority of oligodendrocytes simultaneously transcribe $(x 29, \mathrm{C} \times 32$, and $\mathrm{C} \times 47$. $A$, In heterozygote spinal cord, $\beta$-gal (red) was observed in every CC1-positive (green) cell. Note the small soma consisting of large nucleus (red) and scant cytoplasm (green), typical of oligodendrocytes. $(x 32$-positive $(B)$ and $(x 47-$ positive $(C)$ puncta outline typical $\beta$-gal-positive cells and are distributed along proximal process. Scale bars, $10 \mu \mathrm{m}$.

drocytes and Bergmann glia. In addition, most if not all oligodendrocytes expressed Cx32 and Cx47 in addition to Cx29. As illustrated in Figure 2, $B$ and $C$, macular or punctate $\mathrm{Cx} 32$ and $\mathrm{Cx} 47$ staining was consistently associated with $\beta$-gal-positive cell bodies (Fig. $2 B, C$ ). The small soma and scant cytoplasm of the oligodendrocyte (compare the distribution of $\beta$-gal, which is specifically localized to the nucleus, with $\mathrm{CC} 1$, a soluble protein restricted to the cytoplasm) make it more difficult to distinguish plasma membrane from intracellular membrane connexin. Nev- 
ertheless, the number, size, and distribution of the puncta in Figure 2 suggested gap junctional plaques. Perinuclear (intracellular membrane) connexin staining is generally diffuse and/or composed of very numerous, very small puncta, whereas Cx32 and Cx47 staining consisted of relatively few, large puncta distinctly separated from the nucleus by the extent of the cytoplasm.

The spatial relationship of Cx29 to the other oligodendrocyte connexins has not been explored in detail. Previously, we reported no overlap between Cx29 and $\mathrm{Cx} 32$, but that study used unfixed, conventional cryostat sections of rat spinal cord white matter only (Altevogt et al., 2002). Therefore, we examined the distribution of the three oligodendrocyte connexins by triple staining well fixed (see Materials and Methods) semithin sections $(<1 \mu \mathrm{m})$ of mouse spinal cord (Fig. 3). In all of the following images, cell bodies are identified by $4^{\prime}, 6^{\prime}$-diamidino-2-phenylindole (DAPI) staining, which is pseudocolored white. Cx29 (blue) was much more abundant in gray matter (GM) than in white matter (WM). Cx29 labeling was more diffuse and irregular in shape than labeling for $\mathrm{Cx} 32$ (red) or $\mathrm{Cx} 47$ (green), consistent with the notion that $\mathrm{Cx} 29$ was not generally incorporated into gap junctional plaques. However, glial processes are so complex that cell-cell boundaries are obscured, and it was not possible to determine whether Cx29 was present in gap junctions, nonjunctional plasma membranes, intracellular compartments, or some combination of the three. A gray matter oligodendrocyte cell body was examined at high magnification in the panels at the bottom of the Figure 3. Cx29 (blue) rarely colocalized with either Cx32 (red) or Cx47 (green). In contrast, Cx32 and Cx47 were highly colocalized around oligodendrocyte somata and proximal processes (Fig. 3, yellow) and displayed the characteristic punctate appearance consistent with gap junctional plaques. In the white matter, $\mathrm{Cx} 32$ and $\mathrm{Cx} 47$ also showed some colocalization at oligodendrocyte somata (arrow); however, the degree of coincidence was less than observed in the gray matter. Thus, oligodendrocytes generally express three connexins, but each has a unique distribution. Cx32 and Cx47 display a partial and regionally specific overlap, whereas Cx29 has a completely non-overlapping distribution.

\section{Astrocytes express three connexins forming at least two different classes of gap junctions}

Astrocyte $\mathrm{Cx} 26, \mathrm{Cx} 30$, and $\mathrm{Cx} 43$ also displayed striking regional differences of distribution in spinal cord (Fig. 4A-C). Immunostaining for all three connexins was far more evident in gray matter than in white. Immunofluorescent signals for these connexins were generally punctate in character, suggesting association with a gap junctional plaque. Overall, $\mathrm{Cx} 43\left(1.7 \pm 0.6 / \mu \mathrm{m}^{2}\right)$ and $\mathrm{Cx} 30\left(1.3 \pm 0.6 / \mu \mathrm{m}^{2}\right)$ puncta were more numerous than those containing $\mathrm{Cx} 26\left(0.6 \pm 0.3 / \mu \mathrm{m}^{2}\right)$.

To illustrate the relative distributions of astrocyte connexins, the boxed areas in Figure $4 A-C$ are examined at higher magnification in the panels located on the right. Cx30 (Fig. $4 A$, red) and Cx43 (Fig. 4A, green) displayed extensive overlap (yellow, merged image). Because the ratio of $\mathrm{Cx} 30$ to $\mathrm{Cx} 43$ was variable, some of the signal in the merged image is not yellow. However, careful inspection of the separate channels reveals that the vast majority of puncta contain both connexins (arrows). Cx30 and Cx43 were essentially coincident in both white and gray matter [98.2 $\pm 0.5 \%$ of Cx30-positive puncta also contained Cx43 $(n=$ 481; three separate micrographs); $94.9 \pm 1.3 \%$ of $\mathrm{Cx} 43$-positive puncta also contained $\mathrm{Cx} 30(n=497)]$. In contrast, although occasional colocalization of $\mathrm{Cx} 26$ with $\mathrm{Cx} 30$ and $\mathrm{Cx} 43$ was evident (vide infra), the majority of puncta positive for Cx26 were not positive for either of the other astrocyte connexins [4.0 \pm $1.2 \%$ of Cx26-positive puncta contained Cx30 $(n=202$; three separate micrographs; $6.4 \pm 1.4 \%$ of $\mathrm{Cx} 26$-positive puncta contained $\mathrm{Cx} 43$ ( $n=173)$ ] (Fig. $4 B, C$, merge panels). Therefore, assuming that these puncta represent gap junctional plaques, astrocytes form two classes of gap junction, one containing Cx43 

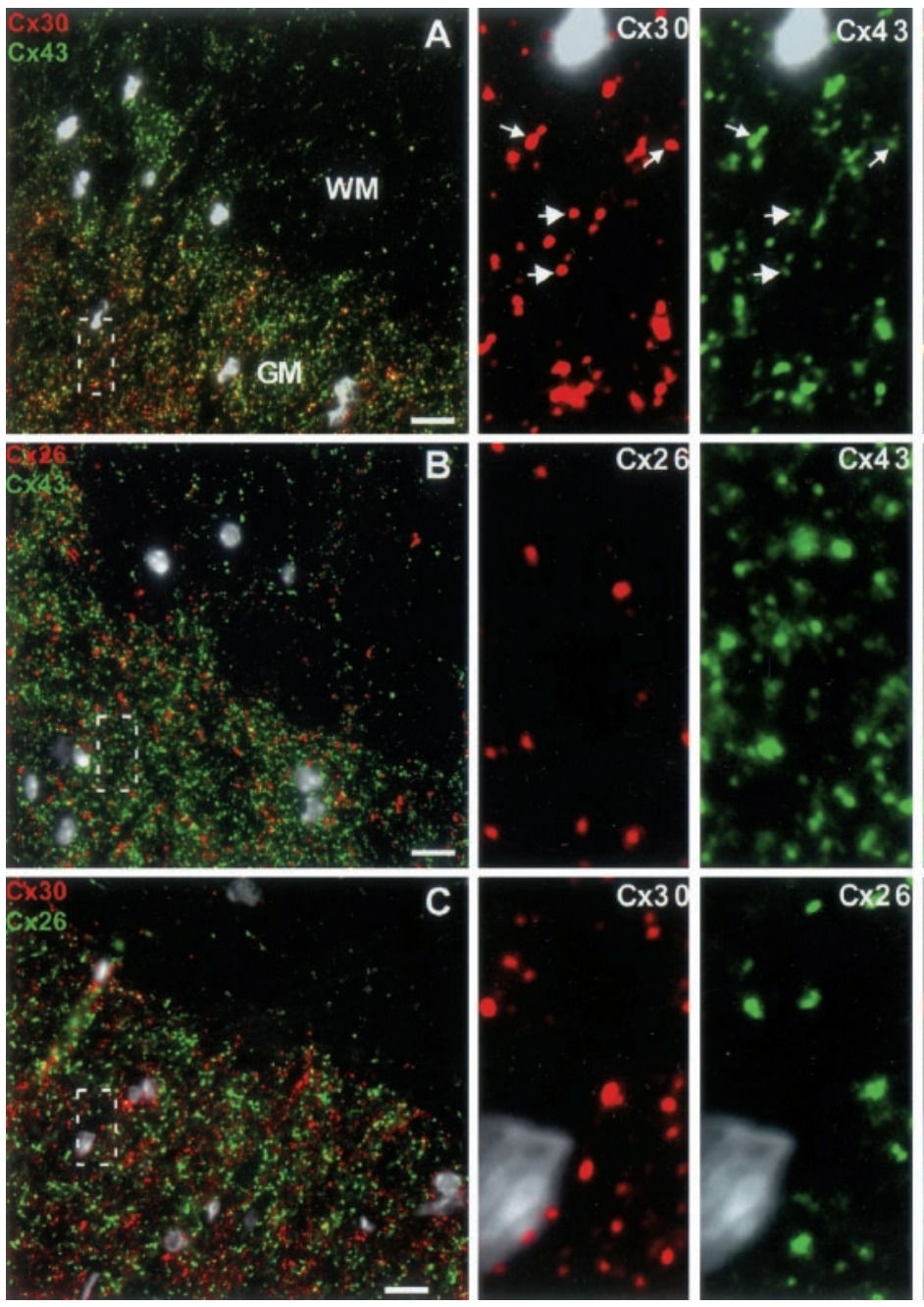

Figure 4. There are at least two classes of astrocyte gap junctions, those containing $C \times 26$ and those containing $C \times 43$ and $C \times 30$. $A$, At low magnification, $\mathrm{C} \times 30$ (red) and $\mathrm{C} 43$ (green) are relatively more abundant in gray matter than in white matter. The boxed area is displayed in the high-magnification panels on the right, which reveal that $C \times 30$ and $C x 43$ are primarily colocalized (yellow). Because the ratio of $\mathrm{Cx} 30$ to $\mathrm{Cx} 43$ was variable, not every puncta in the merged image appears yellow. However, careful inspection of the separate channels reveals that the majority of puncta contain both connexins (arrows). $B$, Alternatively, Cx26 (red) and $\mathrm{Cx} 43$ (green) are mostly not colocalized. In addition, $C \times 43$ puncta are more numerous than those containing Cx26. C, As predicted from the above results, Cx26 (green) and Cx30 (red) rarely colocalize. All nuclei are visualized by DAPI (pseudo-white). Scale bars, $10 \mu \mathrm{m}$.

and $\mathrm{Cx} 30$ and the other containing Cx26. Potentially, one class could be specific for astrocyte-astrocyte communication, whereas the other could provide astrocyte-oligodendrocyte communication. Alternatively, both classes could serve both functions. To discriminate between these possibilities, we used triple labeling to define the spatial relationship between astrocyte and oligodendrocyte connexins.

\section{Astrocyte-oligodendrocyte junctions use specific heterotypic connexin pairings}

Figure 5 compares the spatial relationship of oligodendrocyte Cx29 to astrocyte Cx26 and Cx43, which exemplify the two classes of astrocyte gap junctions. At low power, little overlap in the distributions of any of these connexins is evident in either white or gray matter of the spinal cord. This observation was confirmed by examination of gray matter (boxed area) at higher power in the panels located at the bottom of the figure. Oligodendrocyte Cx29 (red) is almost never associated with either Cx26 (blue) or $\mathrm{Cx} 43$ (green) [0.0\% Cx26-positive puncta also con-
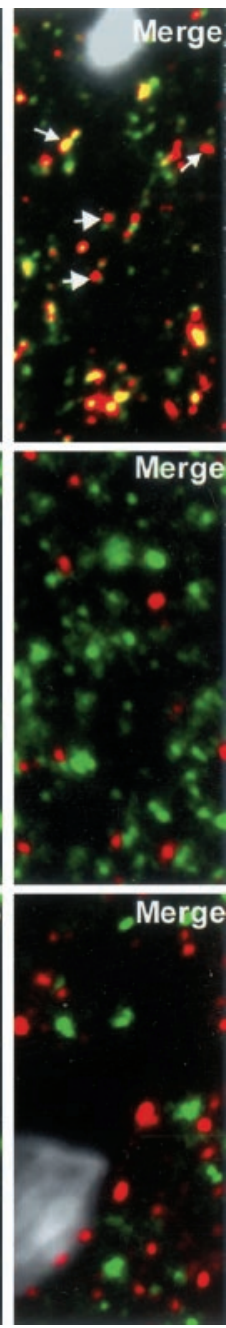

tained Cx29 $(n=53) ; 1.0 \pm 1.2 \% \mathrm{Cx} 43-$ positive puncta also contained Cx29 $(n=$ 189)]. These data suggest that either $C x 29$ does not contribute to astrocyte-oligodendrocyte coupling or interacts with an as yet unidentified astrocyte connexin.

Figure 6 compares the relationship of oligodendrocyte $\mathrm{Cx} 32$ (red) and $\mathrm{Cx} 47$ (green) with astrocyte $\mathrm{Cx} 43$ (blue). At low magnification, it was evident that $\mathrm{Cx} 43$ containing puncta (blue) were much more numerous than those containing the two oligodendrocyte connexins. This likely reflects the relative abundance of astrocyteastrocyte gap junctions compared with astrocyte-oligodendrocyte junctions (Rash et al., 2001). A high-magnification view of an oligodendrocyte cell body in the box marked $A$ is presented at the bottom of the figure. In this typical oligodendrocyte, a high degree of overlap for all three connexins is evident (white puncta, raising the question of whether astrocyte $\mathrm{Cx} 43$ preferentially interacts with one or both of the oligodendrocyte connexins (Cx32 and Cx47). To discriminate between these possibilities, we searched for relatively rare examples in which oligodendrocyte Cx32 and $\mathrm{Cx} 47$ did not overlap. The box marked $B$ encloses one such example in which an oligodendrocyte is closely surrounded with $\mathrm{Cx} 47$ but not $\mathrm{Cx} 32$. Here, most $\mathrm{Cx} 47$ containing puncta that are $\mathrm{C} \times 32$ negative are associated with $\mathrm{Cx} 43$ (91.7 $\pm 2.0 \%$; $n=293$; three micrographs). In contrast, the majority of $\mathrm{Cx} 32$-containing puncta that are $\mathrm{Cx} 47$ negative are not associated with $\mathrm{Cx} 43$ (3.4 $\pm 1.2 \% ; n=147)$. Similar associations were observed at cell bodies and along processes in both white and gray matter. Furthermore, we obtained identical results when comparing the distribution of $\mathrm{C} \times 32$ and $\mathrm{Cx} 47$ to astrocyte $\mathrm{Cx} 30$ (Fig. 7). To determine the oligodendrocyte partner of $\mathrm{Cx} 30$, we again searched for examples in which oligodendrocyte Cx32 and Cx47 did not overlap. A highmagnification view of such an area (box) is presented at the bottom of Figure 7. Overlap of $\mathrm{Cx} 47$ (green) with Cx30 (blue) would be indicated by cyan, whereas overlap of Cx32 (red) with Cx30 (blue) would be indicated by purple. Overall, $91.6 \pm 5.8 \%$ of Cx47-positive, Cx32-negative puncta contained Cx30 ( $n=252$; five micrographs), whereas only $2.6 \pm 1.9 \%$ of $\mathrm{Cx} 32$-positive, $\mathrm{Cx} 47$-negative puncta contained $\mathrm{Cx} 30(n=247)$. Our data are most consistent with a model in which $\mathrm{Cx} 43$ and $\mathrm{Cx} 30$ serve as heterotypic partners for oligodendrocyte $\mathrm{Cx} 47$.

The observation that oligodendrocyte $\mathrm{Cx} 47$ generally associates with astrocyte $\mathrm{Cx} 43 / \mathrm{Cx} 30$ raises the possibility that oligodendrocyte $\mathrm{Cx} 32$ specifically associates with astrocyte $\mathrm{Cx} 26$. This was investigated by triple staining for $\mathrm{Cx} 26, \mathrm{Cx} 32$, and $\mathrm{Cx} 47$ and looking for the relatively rare situations in which $\mathrm{Cx} 32$ and $\mathrm{Cx} 47$ were not colocalized (Fig. 8). As expected, Cx26-containing puncta (green) were far more numerous than either of the two oligodendrocyte connexins, and all three connexins were gener- 
ally associated (white) around oligodendrocyte cell bodies (arrow). However, in the instances in which oligodendrocyte $\mathrm{Cx} 32$ and $\mathrm{Cx} 47$ were not colocalized (box), astrocyte Cx26 was far more often associated with oligodendrocyte $\mathrm{Cx} 32$ $(82.8 \pm 8.4 \% ; n=293)$ than with $\mathrm{Cx} 47$ $(9.8 \pm 2.5 \% ; n=330)$. These data suggest that astrocyte Cx26 preferentially forms heterotypic channels with oligodendrocyte Cx32 instead of Cx47. Thus, we can define at least four classes of glial intercellular channels: two between astrocytes and two between astrocytes and oligodendrocytes (see Discussion).

\section{Distribution of glial connexins in KO animals}

On the basis of the above conclusions, we predicted that loss of Cx29 would not affect the distributions of the other five glial connexins. Triple-labeling studies performed on Cx29 KO spinal cord confirmed this prediction (data not shown). On the other hand, we would predict that loss of Cx32 would cause significant changes in connexin distribution. If astrocyte $\mathrm{Cx} 26$ and oligodendrocyte $\mathrm{Cx} 32$ are obligate heterotypic partners, and if Cx32 were not available, then Cx26 should not accumulate around oligodendrocyte cell bodes as it normally does. The first column in Figure 9 compares the distributions of
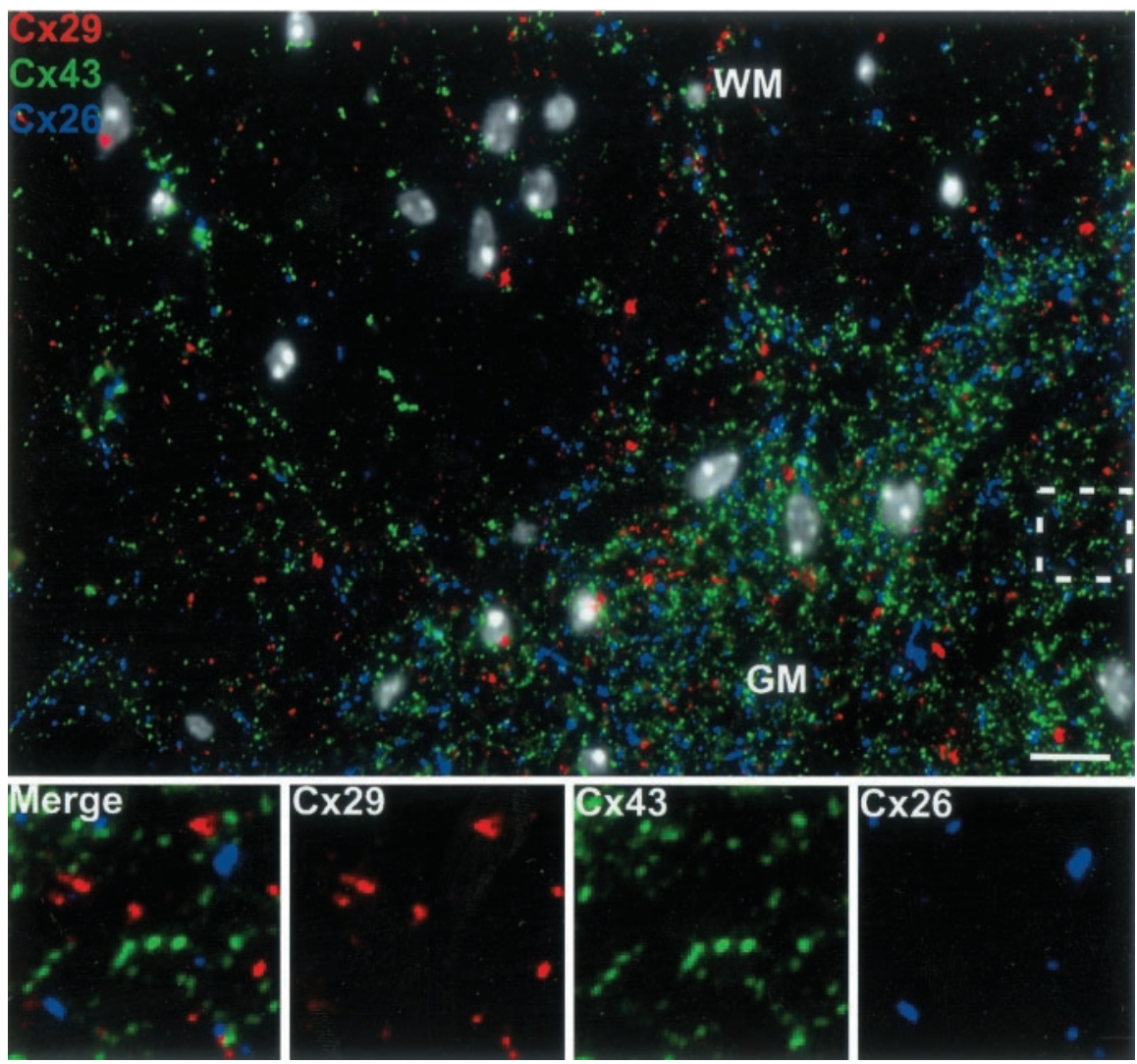

Figure 5. Oligodendrocyte (x29 does not associate with any astrocyte connexin. There is no obvious overlap between oligodendrocyte Cx29 (red) and astrocyte (x26 (blue) or Cx43 (green) at low magnification in any region of the spinal cord. This is confirmed at higher magnification of the boxed area. All nuclei are visualized by DAPI (pseudo-white). GM, Gray matter; WM, white matter. Scale bar, $10 \mu \mathrm{m}$.

astrocyte Cx26 in WT and Cx32 KO spinal

cord. Cx47 (green) was used as a marker to identify oligodendrocyte cell bodies (Menichella et al., 2003). As predicted, the association of astrocyte Cx26 (red) with oligodendrocyte cell bodies in the WT (yellow) was lost in the Cx32 KO.

If astrocyte $\mathrm{Cx} 43$ and $\mathrm{Cx} 30$ are obligate heterotypic partners for oligodendrocyte $\mathrm{Cx} 47$, then their distributions should be unaltered in the absence of $\mathrm{Cx} 32$. Indeed, colocalization of $\mathrm{Cx} 43$ (red) and Cx47 (green) at oligodendrocyte cell bodies was unaffected in the Cx32 KO (Fig. 9, second column, yellow). However, astrocyte $\mathrm{Cx} 30$ (red) and oligodendrocyte $\mathrm{Cx} 47$ (green) were no longer associated in the $\mathrm{KO}$ (Fig. 9, third column). The general coincidence of $\mathrm{Cx} 30$ and $\mathrm{Cx} 43$ is not affected in the $\mathrm{KO}$ (data not shown), only their colocalization around oligodendrocyte soma. One explanation for this result is that $\mathrm{Cx} 30$ and $\mathrm{Cx} 32$ are heterotypic partners. However, Cx30 does not colocalize with $\mathrm{Cx} 32$ in WT spinal cord (Fig. 8). Therefore, we propose that loss of $\mathrm{Cx} 30$ at oligodendrocyte cell bodies in the $\mathrm{KO}$ most likely represents a compensatory change attributable to the absence of $\mathrm{Cx} 26 / \mathrm{Cx} 32$ heterotypic channels (see Discussion).

\section{Discussion}

Our data suggest that astrocytes form two distinct classes of gap junctions with each other: one containing Cx26 (Fig. 10a) and a second containing $\mathrm{Cx} 43$ and $\mathrm{Cx} 30$ (Fig. 10b,c). This notion is consistent with in vitro expression studies demonstrating that Cx26 forms neither heteromeric channels with Cx43 (Beyer et al., 2001) nor heterotypic channels with Cx30 (Manthey et al., 2001). If $\mathrm{Cx} 30$ and $\mathrm{Cx} 43$ interact to form heteromeric connexons, which has not yet been demonstrated, then the second class of astrocyte gap junction would consist primarily of heteromeric, heterotypic intercellular channels (Fig. 10b). If Cx30 and Cx43 do not form heteromeric connexons, then the second class of astrocyte gap junction might contain multiple types of intercellular channel (Fig. 10c). Our findings also suggest an unexpected complexity for gap junctions between astrocytes and oligodendrocytes. These gap junctions are not separated into obligatory classes. However, it appears that all astrocyte-oligodendrocyte gap junctions contain heterotypic channels. Our data suggest that astrocyte $\mathrm{Cx} 26$ associates mainly with oligodendrocyte $\mathrm{Cx} 32$, whereas astrocyte $\mathrm{Cx} 43$ and $\mathrm{Cx} 30$ associate with oligodendrocyte $\mathrm{Cx} 47$. If, as illustrated in Figure 10d, Cx30 and Cx43 form heteromeric connexons, then two types of astrocyte-oligodendrocyte intercellular channels are expected. If they do not, then three or four different types might be encountered. Regardless, our data provide the first direct evidence for the segregation of astrocyte connexins into different locations and the existence of naturally occurring heterotypic channels.

The relative abundance of immunoreactive puncta in the gray matter relative to the white matter is reasonably consistent with other studies of astrocyte connexin distribution using in situ hybridization, immunocytochemistry, or cell fractionation (Nagy et al., 1999; Lynn et al., 2001; Condorelli et al., 2002). It is not clear whether this results from regional differences in gene expression, in astrocyte number, or both. In vivo, morphological differences between white matter (fibrous) and gray matter (protoplasmic) astrocytes have been well established in mammalian species, supporting the notion of regional differences in gene expression. Subpopulations of astrocytes have also been defined 

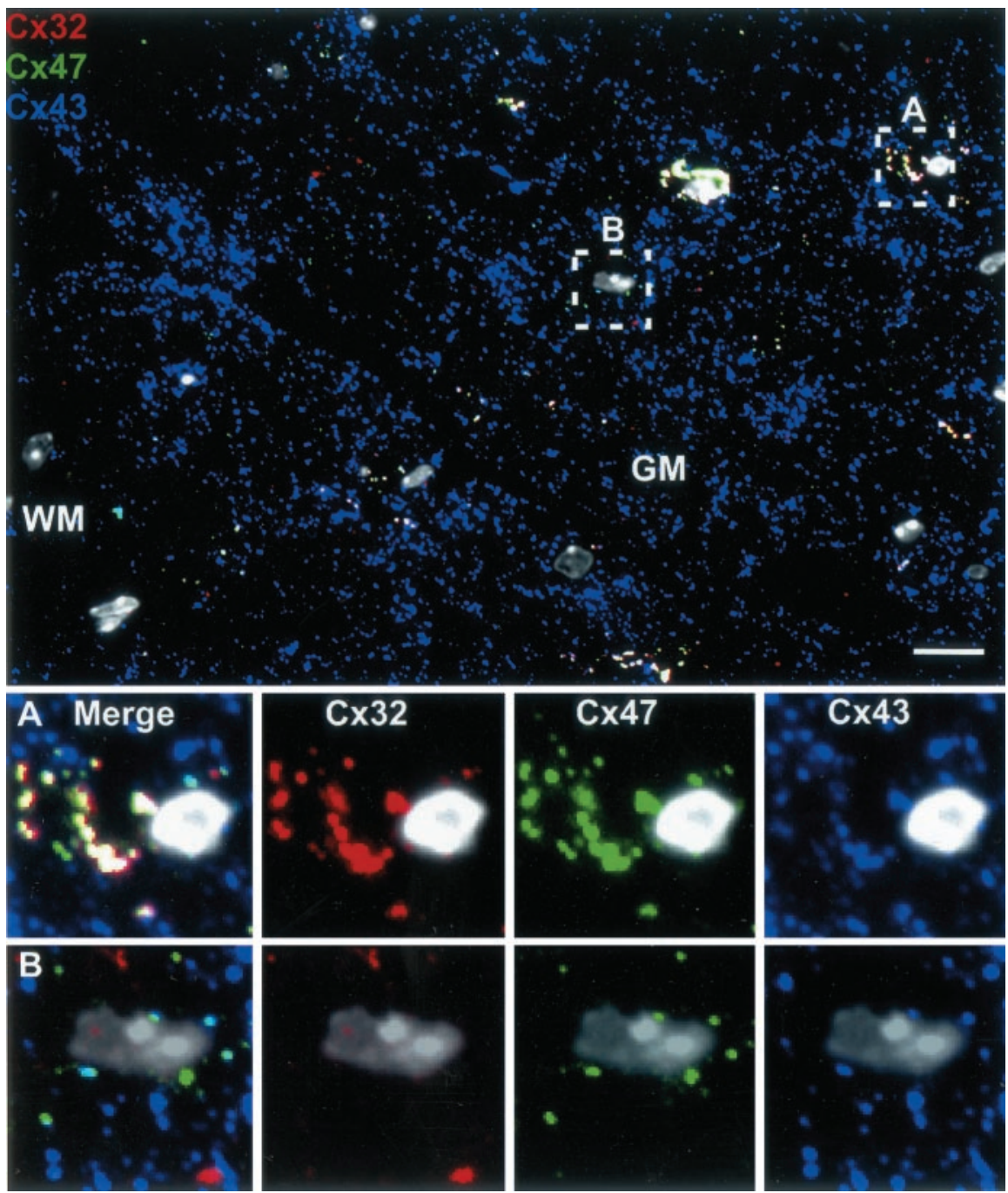

Figure 6. Astrocyte Cx43 preferentially associates with oligodendrocyte Cx47. Triple labeling for Cx32 (red), Cx43 (blue), and Cx47 (green) in adult WT spinal cord demonstrates a high degree of overlap of all three connexins (white puncta) at oligodendrocyte cell bodies and proximal processes in the gray matter. Box $A$ illustrates a typical oligodendrocyte cell body in which all three connexins are colocalized. Box B illustrates a rare oligodendrocyte cell body in which Cx47 and Cx32 do not colocalize. In these circumstances, Cx43 associates only with Cx47 (cyan) and not with Cx32. All nuclei are visualized by DAPI (pseudo-white). Scale bar, $10 \mu \mathrm{m}$.

in culture [type 1 and type 2 (Raff et al., 1983)]. Strikingly, type 2 astrocytes neither establish functional intercellular channels with each other nor express Cx26, Cx32, or Cx43 (Sontheimer et al., 1991; Belliveau and Naus, 1994). Although the relationship of type 2 astrocytes to fibrous white matter astrocytes is not clear, the presence of two astrocyte subpopulations in culture is at least consistent with the notion of regional difference in astrocyte gene expression. Thus, the regional difference in connexin levels may reflect a difference in metabolic requirements of astrocytes in white and gray matter. On the other hand, connexin levels may reflect a difference in the numbers of white matter and gray matter astrocytes. In support of this notion, white matter contains far fewer astrocytes than gray matter in zebrafish (Kawai et al., 2001) and bullfrog (Sasaki and Mannen, 1981) spinal cord. However, all direct comparisons of cell numbers have been hampered by the lack of a simple method by which all astrocytes can be unequivocally identified.

One unexpected finding was that $\mathrm{Cx} 29$ rarely colocalized with any of the other five glial connexins. Cx29 staining cannot corre- spond to gap junctions between adjacent oligodendrocytes, because gap junctions between these cells are rarely if ever observed (Massa and Mugnaini, 1982; Rash et al., 2001). Because we cannot conclusively establish whether Cx29 signal arises from plasma membranes using morphologic criteria, it is possible that $\mathrm{Cx} 29$ is actually restricted to intracellular membranes. A second possibility is that $\mathrm{Cx} 29$ forms heterotypic astrocyte-oligodendrocyte junctions involving an as yet unidentified connexin. To address this issue, we performed reverse transcription-PCR on microdissected spinal cord white matter and detected several additional connexin transcripts (data not shown). It will be of interest to determine their cellular origin. A third possibility is that $\mathrm{Cx} 29$ does not form intercellular channels but forms connexons that display channel activity in a single plasma membrane. Such "hemichannel" activity has been reported for a number of connexins, including Cx43 ( $\mathrm{Li}$ et al., 1996) and Cx32 (Castro et al., 1999), and in a number of different cell types, including astrocytes (Stout et al., 2002; Ye et al., 2003). Although Cx29 hemichannel activity has not yet been reported, its subcellular distribution in Schwann cells (Altevogt et al., 2002) is most consistent with a hemichannel function allowing glial uptake of $\mathrm{K}^{+}$from the small, private extracellular space between axon and Schwann cell (Brophy, 2001).

Some of our findings conflict with reports in which extensive colocalization of Cx43/Cx30 with Cx26 was observed using light microscopic immunocytochemistry (Nagy et al., 2001). Although the origins of this discrepancy are not clear, there were several substantive differences in the experimental approaches. First, our study was focused exclusively on the spinal cord, which was not examined using light microscopy in the previous study. Second, we used semithin $(<1 \mu \mathrm{m})$ frozen sections, whereas the previous study used conventional $10 \mu \mathrm{m}$ cryostat sections and confocal microscopy. Third, our fixation protocol included glutaraldehyde, which dramatically improved tissue morphology compared with fixation with formaldehyde alone, as assessed by bright-field microscopy of histologically stained specimens (data not shown). Surprisingly, the majority of our anti-connexin antibodies could be used successfully on semithin sectioned, glutaraldehyde-fixed materials. Fourth, accurate assessment of the relationships between multiple connexins was facilitated in our study by triple immunolabeling, which has not been performed previously. Furthermore, the use of semithin sections allowed the connexin distributions to be analyzed in the same area of the cord, minimizing any problem of regional variability attributable to sample preparation or other extrinsic factors.

A study by Nagy and colleagues (Lynn et al., 2001) also included freeze-fracture electron microscopic immunolabeling 
(FRIL) demonstrating colocalization of Cx30 and Cx26 in gap junctions between astrocytes in rat spinal cord, which is not consistent with the patterns of staining we observed using light microscopy. One explanation may reflect a difference in sensitivity between the labeling procedures. For example, in their micrograph displaying an astrocyte-astrocyte gap junction, FRIL labeling for $\mathrm{Cx} 30$ consists of a single gold particle, whereas Cx26 is represented by numerous particles. On the other hand, FRIL labeling of gap junctions between astrocytes and oligodendrocytes contain equal numbers of particles representing both connexins, which is consistent with our data. Thus, Cx30 may be a minor component of astrocyte-astrocyte junctions containing $\mathrm{Cx} 26$, reconciling the discrepancy between the FRIL study and our results.

In addition to the conflicts discussed above, a recent study reported that CNS expression of Cx26 was limited exclusively to meningeal cells (Filippov et al., 2003). This study noted an absence of marker expression in the embryonic brain parenchyma of $\mathrm{Cx} 26-\beta$-galactosidase knock-in mice and a lack of Cx26 in situ hybridization signal in the adult brain of WT mice. However, transcription from the modified allele was severely reduced, which would preclude the detection of all but the strongest signals. In this regard, using immunocytochemistry, Mercier and Hatton (2001)
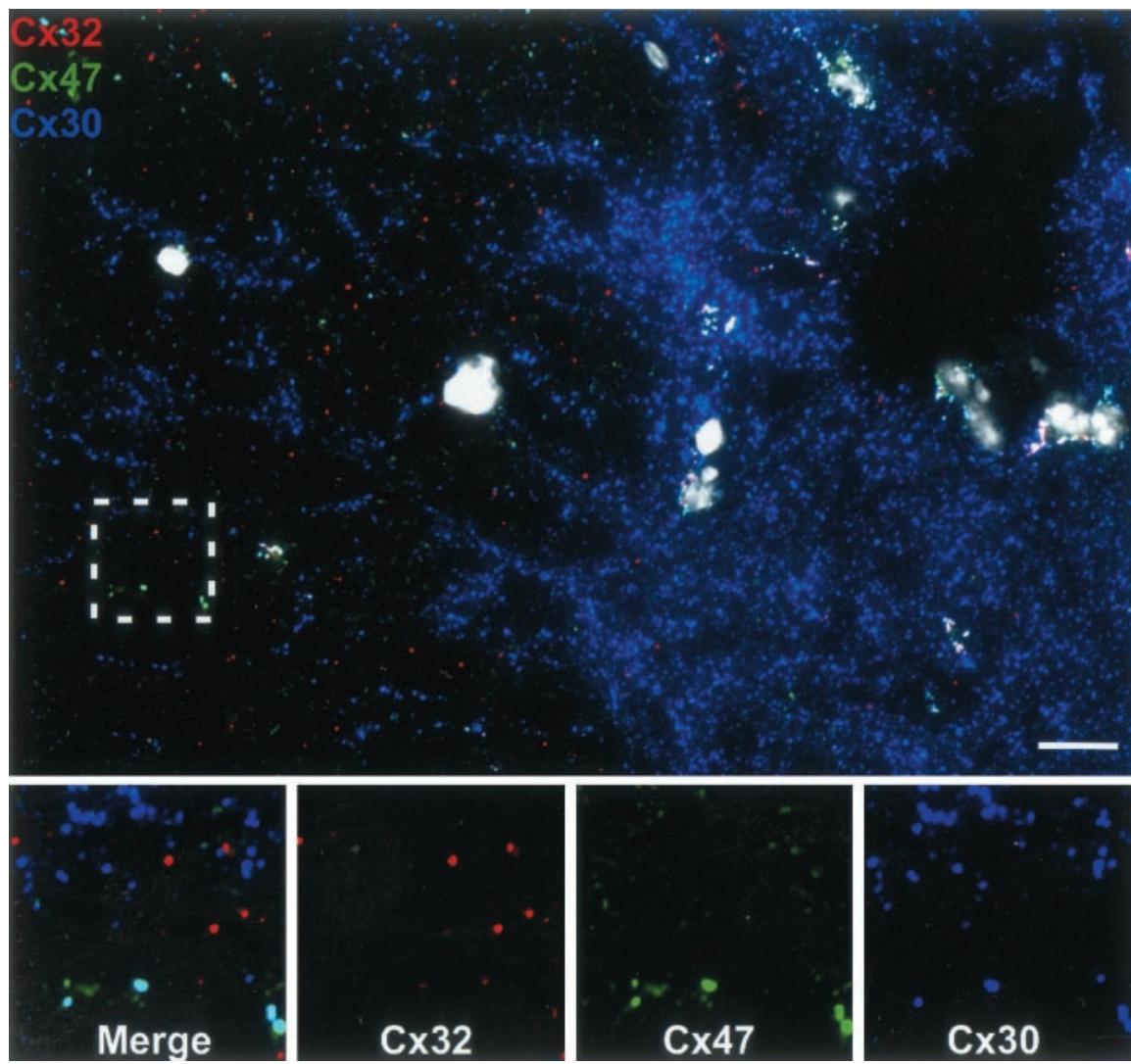

Figure 7. Astrocyte $\mathrm{C} \times 30$ preferentially associates with oligodendrocyte $\mathrm{C} \times 47$. Triple labeling for $\mathrm{C} \times 32$ (red), $\mathrm{C} \times 30$ (blue), and Cx47 (green) adult WT spinal cord demonstrates a high degree of overlap of all three connexins (white puncta) at oligodendrocyte cell bodies and proximal processes in the gray matter. To determine the oligodendrocyte partner of $\mathrm{Cx} 30$, we searched for examples in which oligodendrocyte $\mathrm{Cx} 32$ and $\mathrm{Cx} 47$ did not overlap (box). Extensive overlap of $\mathrm{Cx} 47$ (green) with $\mathrm{Cx} 30$ (blue) was observed (cyan), whereas $\mathrm{Cx} 32$ (red) was unassociated with $\mathrm{Cx} 30$. All nuclei are visualized by DAPI (pseudowhite). Scale bar, $10 \mu \mathrm{m}$. found Cx26 much more abundant in subependymal, subpial, and perivascular zones containing leptomeninges but still found Cx26 in GFAP-positive astrocytes throughout the brain parenchyma. Furthermore, Filippov et al. (2003) did not examine marker expression in adult spinal cord, the area of concentration in our study. Thus, regional variation in connexin expression might also contribute to the discrepancies between Filippov et al. and the studies using immunocytochemistry. In fact, regional variation in $\mathrm{Cx} 26$ levels was reported previously in astrocytes (Nagy et al., 2001). For our study, antibody staining was validated by using two different commercially prepared anti-Cx26 antibodies, a mouse monoclonal and an affinity-purified rabbit anti-peptide antibody, which gave similar results. However, we cannot rule out the possibility that both preparations of antibodies produce similar artifacts.

The distribution of glial connexins in WT spinal cord suggested the existence of four or more distinct classes of intercellular channels (Fig. 10), a notion that was tested by examination of connexin distribution in the $\mathrm{Cx} 32 \mathrm{KO}$. Our model suggested that the colocalization of $\mathrm{Cx} 47$ and $\mathrm{Cx} 26$ at oligodendrocyte cell bodies (Fig. 8, low magnification) actually reflects the heterotypic association of oligodendrocyte Cx47 with astrocyte Cx43/Cx30 and of oligodendrocyte $\mathrm{Cx} 32$ with astrocyte Cx26. If true, then Cx26 should no longer be associated with oligodendrocyte cell bodies in animals lacking $\mathrm{Cx} 32$. Indeed, association of $\mathrm{Cx} 26$ with oligodendrocyte cell bodies (defined by the presence of $\mathrm{Cx} 47$ positive puncta) was lost in the Cx32 KO. Our model is further supported by in vitro expression studies demonstrating that $\mathrm{C} \times 32$

forms functional heterotypic intercellular channels with Cx26 (Rubin et al., 1992; Bukauskas et al., 1995).

Another prediction was that the association of $\mathrm{Cx} 43 / \mathrm{Cx} 30$ with $\mathrm{Cx} 47$ should be undisturbed in the $\mathrm{Cx} 32 \mathrm{KO}$. This was true for $\mathrm{Cx} 43$, but, unexpectedly, it was not true for $\mathrm{Cx} 30$. The redistribution of both $\mathrm{Cx} 26$ and $\mathrm{Cx} 30$ in the $\mathrm{Cx} 32 \mathrm{KO}$ are similar to those reported recently by Nagy et al. (2003). Importantly, the $\mathrm{Cx} 32 \mathrm{KO}$ did not exhibit changes in the expression levels of the other glial connexins (data not shown). In addition, the dissociation of $\mathrm{Cx} 43$ and $\mathrm{Cx} 30$ was limited specifically to gap junctions located near oligodendrocyte cell bodies, presumably gap junctions between astrocytes and oligodendrocytes, whereas the general association of $\mathrm{Cx} 43$ and $\mathrm{Cx} 30$, presumably representing astrocyte-astrocyte gap junctions, was not changed. One interpretation, favored by Nagy et al. (2003), is that astrocyte Cx30 may interact with oligodendrocyte Cx32 to form heterotypic channels. Functional heterotypic interactions between these connexins have been observed in vitro (Dahl et al., 1996). However, in WT spinal cord, we did not find Cx30 colocalized with $\mathrm{Cx} 32$ except in the presence of $\mathrm{Cx} 47$ (Fig. 8), arguing against a heterotypic $\mathrm{Cx} 30 / \mathrm{Cx} 32$ interaction in vivo. An alternative explanation is that the loss of heterotypic Cx26-Cx32 intercellular channels in the $\mathrm{Cx} 32 \mathrm{KO}$ is compensated by the specific exclusion of $\mathrm{Cx} 30$ from astrocyte-oligodendrocyte gap junctions. If so, the remaining astrocyte-oligodendrocyte channels (heterotypic Cx43-Cx47) should exhibit properties of conductance and/or permselectivity different from $\mathrm{Cx} 30 / \mathrm{Cx} 43-\mathrm{Cx} 47$ channels. It will 

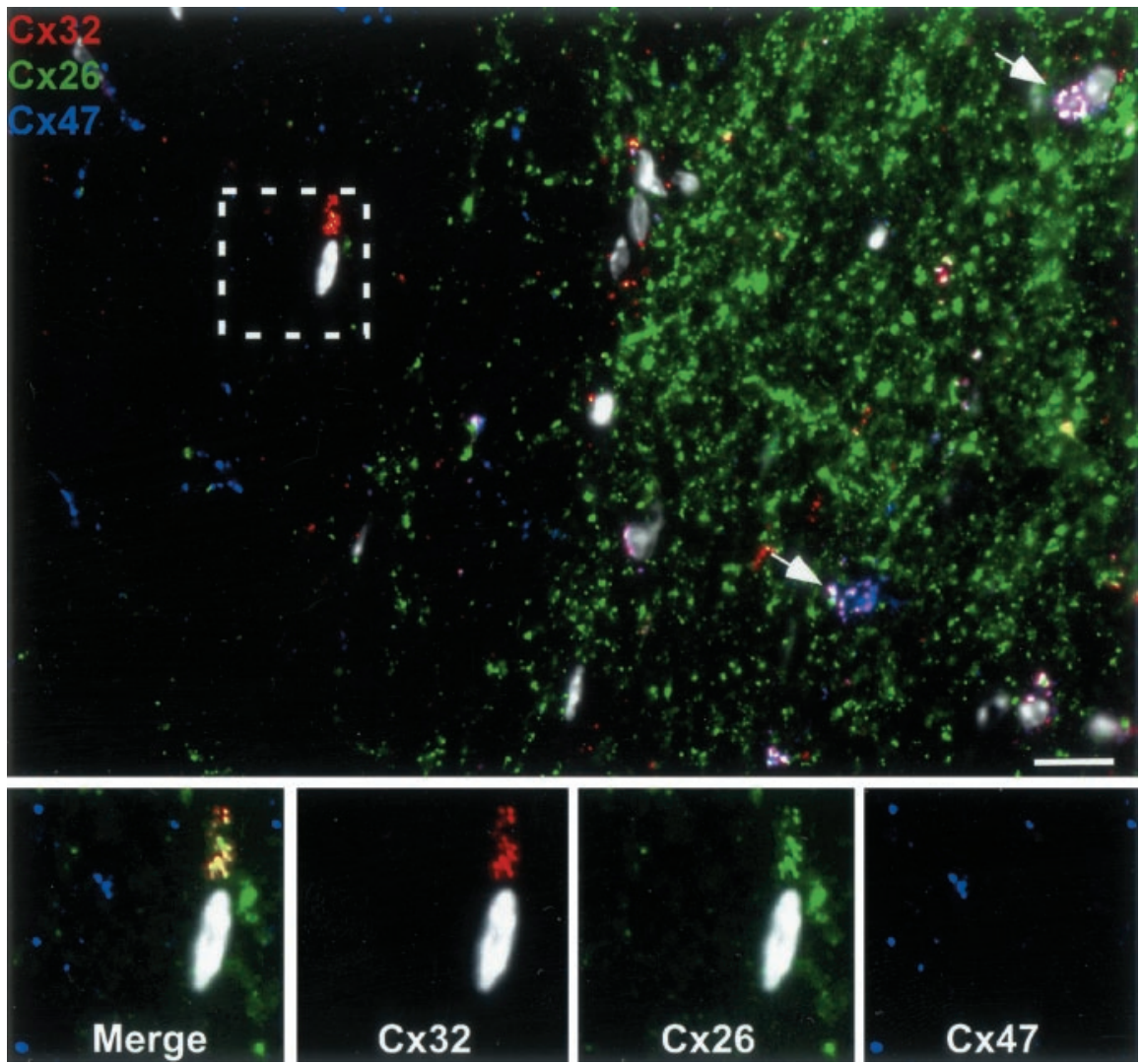

Figure 8. Astrocyte $C x 26$ preferentially associates with oligodendrocyte $C x 32$. Triple labeling for $(x 32$ (red), $C \times 26$ (green), and Cx47 (blue) demonstrates a high degree of overlap of all three connexins (white puncta) at gray matter (GM) oligodendrocyte cell bodies (white arrows) and proximal processes. However, in the white matter (WM), oligodendrocyte $(x 32$ and $C \times 47$ are less often colocalized than in gray matter. In these instances, astrocyte $\mathrm{Cx} 26$ (boxed area) is always associated with oligodendrocyte $\mathrm{Cx} 32$ (yellow) but not with oligodendrocyte Cx47. All nuclei are visualized by DAPI (pseudo-white). Scale bar, $10 \mu \mathrm{m}$.
Balice-Gordon RJ, Bone LJ, Scherer SS (1998) Functional gap junctions in the Schwann cell myelin sheath. J Cell Biol 142:1095-1104.

Belliveau DJ, Naus CCG (1994) Cortical type 2 astrocytes are not dye coupled nor do they express the major gap junction genes found in the central nervous system. Glia 12:24-34.

Bergoffen J, Scherer SS, Wang S, Scott MO, Bone LJ, Paul DL, Chen K, Lensch MW, Chance PF, Fischbeck KH (1993) Connexin mutations in $\mathrm{X}$-linked Charcot-Marie-Tooth disease. Science 262:2039-2042.

Beyer EC, Gemel J, Martinez A, Berthoud VM, Valiunas V, Moreno AP, Brink PR (2001) Heteromeric mixing of connexins: compatibility of partners and functional consequences. Cell Commun Adhes 8:199-204.

Bhat RV, Axt KJ, Fosnaugh JS, Smith KJ, Johnson KA, Hill DE, Kinzler KW, Baraban JM (1996) Expression of the APC tumor suppressor protein in oligodendroglia. Glia 17:169-174.

Brophy PJ (2001) Axoglial junctions: separate the channels or scramble the message. Curr Biol 11:R555-R557.

Bukauskas FF, Elfgang C, Willecke K, Weingart R (1995) Heterotypic gap junctions channels (connexin26-connexin32) violate the paradigm of unitary conductance. Pflügers Arch 430:870-872.

Castejon OJ, Dailey ME, Apkarian RP, Castejon HV (2002) Correlative microscopy of cerebellar Bergmann glial cells. J Submicrosc Cytol Pathol 34:131-142.

Castro C, Gomez-Hernandez JM, Silander K, Barrio LC (1999) Altered formation of hemichannels and gap junction channels caused by C-terminal connexin-32 mutations. J Neurosci 19:37523760.

Celio MR, Heizmann CW (1981) Calciumbinding protein parvalbumin as a neuronal marker. Nature 293:300-302.

be of interest to closely examine the physiological properties of junctions formed from biologically relevant combinations of glial connexins.

CNS glia are unusual in the number of connexins expressed and the complexity of their distribution. What is the purpose of such complexity? One possibility is functional redundancy. In support of this idea, a loss of an individual oligodendrocyte connexin does not cause a dramatic central phenotype [Cx47 (Menichella et al., 2003; Odermatt et al., 2003), Cx32 (Sutor et al., 2000; Kleopa et al., 2002), and Cx29 (this study)], whereas loss of two connexins causes severe demyelination and early mortality [Cx32 and Cx47 (Menichella et al., 2003; Odermatt et al., 2003)]. However, a requirement for redundancy does not explain why astrocytes and oligodendrocyte express non-overlapping sets of three connexins. A clue may come from our observation that $\mathrm{Cx} 30$ is selectively lost from astrocyte-oligodendrocyte gap junctions in the $\mathrm{Cx} 32 \mathrm{KO}$, which could reflect a compensatory adaptation to the loss of $\mathrm{Cx} 26-\mathrm{Cx} 32$ heterotypic channels. The availability of additional connexins may provide flexibility in achieving relatively subtle alterations in the properties of intercellular channels, necessary for maintaining the glial network.

\section{References}

Altevogt BM, Kleopa KA, Postma FR, Scherer SS, Paul DL (2002) Connexin29 is uniquely distributed within myelinating glial cells of the central and peripheral nervous systems. J Neurosci 22:6458-6470.

Alvarez-Maubecin V, Garcia-Hernandez F, Williams JT, Van Bockstaele EJ (2000) Functional coupling between neurons and glia. J Neurosci 20:4091-4098.
Condorelli D, Mudo G, Trovato-Salinaro A, Mirone M, Amato G, Belluardo N (2002) Connexin-30 mRNA is up-regulated in astrocytes and expressed in apoptotic neuronal cells of rat brain following kainate-induced seizures. Mol Cell Neurosci 21:94-113.

Dahl E, Manthey D, Chen Y, Schwarz HJ, Chang YS, Lalley PA, Nicholson BJ, Willecke K (1996) Molecular cloning and functional expression of mouse connexin-30, a gap junction gene highly expressed in adult brain and skin. J Biol Chem 271:17903-17910.

Dermietzel R, Spray DC (1993) Gap junctions in the brain: where, what type, how many and why? Trends Neurosci 16:186-192.

Dermietzel R, Traub O, Hwang TK, Beyer E, Bennett MVL, Spray DC, Willecke K (1989) Differential expression of three gap junction proteins in developing and mature brain tissue. Proc Natl Acad Sci USA 86:10148-10152.

Evans WH, Martin PE (2002) Gap junctions: structure and function. Mol Membr Biol 19:121-136.

Filippov MA, Hormuzdi SG, Fuchs EC, Monyer H (2003) A reporter allele for investigating connexin 26 gene expression in the mouse brain. Eur J Neurosci 18:3183-3192.

Gabriels JE, Paul DL (1998) Connexin43 is highly localized to sites of disturbed flow in rat aortic endothelium but connexin 37 and connexin 40 are more uniformly distributed. Circ Res 83:636-643.

Giaume C, Fromaget C, El Aoumari A, Cordier J, Glowinski J, Gros D (1991) Gap junctions in cultured astrocytes: single-channel currents and characterization of channel-forming protein. Neuron 6:133-143.

Goodenough DA, Paul DL (2003) Beyond the gap: functions of unpaired connexon channels. Nat Rev Mol Cell Biol 4:285-295.

Haydon PG (2001) GLIA: listening and talking to the synapse. Nat Rev Neurosci 2:185-193.

Kawai H, Arata N, Nakayasu H (2001) Three-dimensional distribution of astrocytes in zebrafish spinal cord. Glia 36:406-413. 
Kleopa KA, Yum SW, Scherer SS (2002) Cellular mechanisms of connexin 32 mutations associated with CNS manifestations. J Neurosci Res 68:522-534.

Kunzelmann P, Schroder W, Traub O, Steinhauser C, Dermietzel R, Willecke K (1999) Late onset and increasing expression of the gap junction protein connexin 30 in adult murine brain and long-term cultured astrocytes. Glia 25:111-119.

Li HY, Liu TF, Lazrak A, Peracchia C, Goldberg GS, Lampe PD, Johnson RG (1996) Properties and regulation of gap junctional hemichannels in the plasma membranes of cultured cells. J Cell Biol 134:1019-1030.

Lynn BD, Rempel JL, Nagy JI (2001) Enrichment of neuronal and glial connexins in the postsynaptic density subcellular fraction of rat brain. Brain Res 898:1-8.

Makowski L, Caspar DLD, Phillips WC, Goodenough DA (1977) Gap junction structures. II. Analysis of the x-ray diffraction data. J Cell Biol 74:629-645.

Manthey D, Banach K, Desplantez T, Lee CG, Kozak CA, Traub O, Weingart R, Willecke K (2001) Intracellular domains of mouse connexin 26 and -30 affect diffusional and electrical properties of gap junction channels. J Membr Biol 181:137-148.

Massa PT, Mugnaini E (1982) Cell junctions and intramembrane particles of astrocytes and oligodendrocytes: a freeze-fracture study. Neuroscience 7:523-538.

Menichella DM, Goodenough DA, Sirkowski E, Scherer SS, Paul DL (2003) Connexins are critical for normal myelination in the CNS. J Neurosci 23:5963-5973.

Mercier F, Hatton GI (2001) Connexin 26 and basic fibroblast growth factor are expressed primarily in the subpial and subependymal layers in adult brain parenchyma: roles in stem cell proliferation and morphological plasticity? J Comp Neurol 431:88-104.

Nagy JI, Patel D, Ochalski PA, Stelmack GL (1999) Connexin30 in rodent, cat and human brain: selective expression in gray matter astrocytes, co-localization with connexin 43 at gap junctions and late developmental appearance. Neuroscience 88:447-468.

Nagy JI, Li X, Rempel J, Stelmack G, Patel D, Staines WA, Yasumura T, Rash JE (2001) Connexin26 in adult rodent central nervous system: demonstration at astrocytic gap junctions and colocalization with connexin 30 and connexin43. J Comp Neurol 441:302-323.

Nagy JI, Ionescu AV, Lynn BD, Rash JE (2003) Connexin 29 and connexin 32 at oligodendrocyte and astrocyte gap junctions and in myelin of the mouse central nervous system. J Comp Neurol 464:356-370.

Nedergaard M (1994) Direct signaling from astrocytes to neurons in cultures of mammalian brain cells. Science 263:1768-1771.

Odermatt B, Wellershaus K, Wallraff A, Seifert G, Degen J, Euwens C, Fuss B, Bussow H, Schilling K, Steinhauser C, Willecke K (2003) Connexin 47 (Cx47)-deficient mice with enhanced green fluorescent protein reporter gene reveal predominant oligodendrocytic expression of $\mathrm{Cx} 47$ and display vacuolized myelin in the CNS. J Neurosci 23:4549-4559.

Orkand RK, Nicholls JG, Kuffler SW (1966) Effect of nerve impulses on the membrane potential of glial cells in the central nervous system of amphibia. J Neurophysiol 29:788-806.

Raff MC, Miller RH, Noble M (1983) A glial progenitor cell that develops in vitro into an astrocyte or an oligodendrocyte depending on culture medium. Nature 303:390-396.
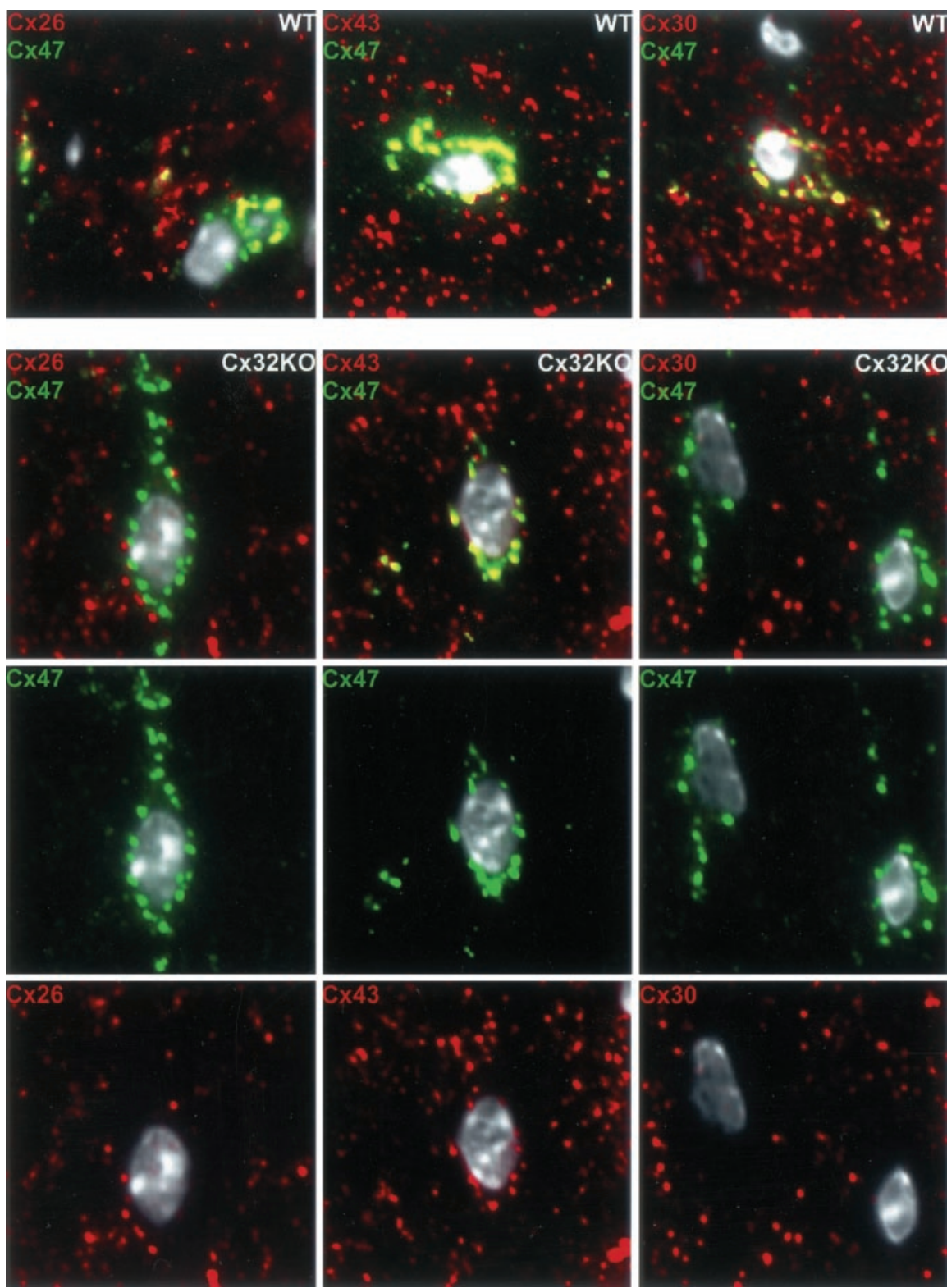

Figure 9. Redistribution of astrocyte connexins at oligodendrocyte cell bodies in $\mathrm{C} 32 \mathrm{~K} 0$ mice. Our data suggest that colocalization of $\mathrm{C} \times 47$ and $\mathrm{C} \times 26$, observed at oligodendrocyte cell bodies (Fig. 7, low magnification), reflects the heterotypic association of $\mathrm{C} 47$ with $\mathrm{C} \times 43 / \mathrm{C} \times 30$ and of $\mathrm{C} \times 26$ with $\mathrm{C} \times 32$. If so, then $\mathrm{C} \times 26$ should no longer be colocalized with $\mathrm{C} \times 47$ at oligodendrocyte cell ( $x 32 \mathrm{KO}$. Indeed, colocalization (yellow) of $\mathrm{Cx} 26$ (red) and $\mathrm{Cx} 47$ (green) (column 1, WT) is lost in the $\mathrm{Cx} 32 \mathrm{KO}$ (column Cx32 K0, indicating that $\mathrm{C} \times 43$ and $\mathrm{C} \times 30$ are no longer heteromeric partners (see Results). All nuclei are visualized by DAPI (pseudo-white). Scale bar, $10 \mu \mathrm{m}$.

Rash JE, Staines WA, Yasumura T, Patel D, Furman CS, Stelmack GL, Nagy JI (2000) Immunogold evidence that neuronal gap junctions in adult rat brain and spinal cord contain connexin-36 but not connexin-32 or connexin-43. Proc Natl Acad Sci USA 97:7573-7578.

Rash JE, Yasumura T, Dudek FE, Nagy JI (2001) Cell-specific expression of connexins and evidence of restricted gap junctional coupling between glial cells and between neurons. J Neurosci 21:1983-2000.

Rubin JB, Verselis VK, Bennett MVL, Bargiello TA (1992) Molecular analysis of voltage dependence of heterotypic gap junctions formed by connexins 26 and 32. Biophys J 62:183-193.

Sasaki H, Mannen H (1981) Morphological analysis of astrocytes in the bullfrog (Rana catesbeiana) spinal cord with special reference to the site of attachment of their processes. J Comp Neurol 198:13-35.

Scherer SS, Deschenes SM, Xu YT, Grinspan JB, Fischbeck KH, Paul DL 

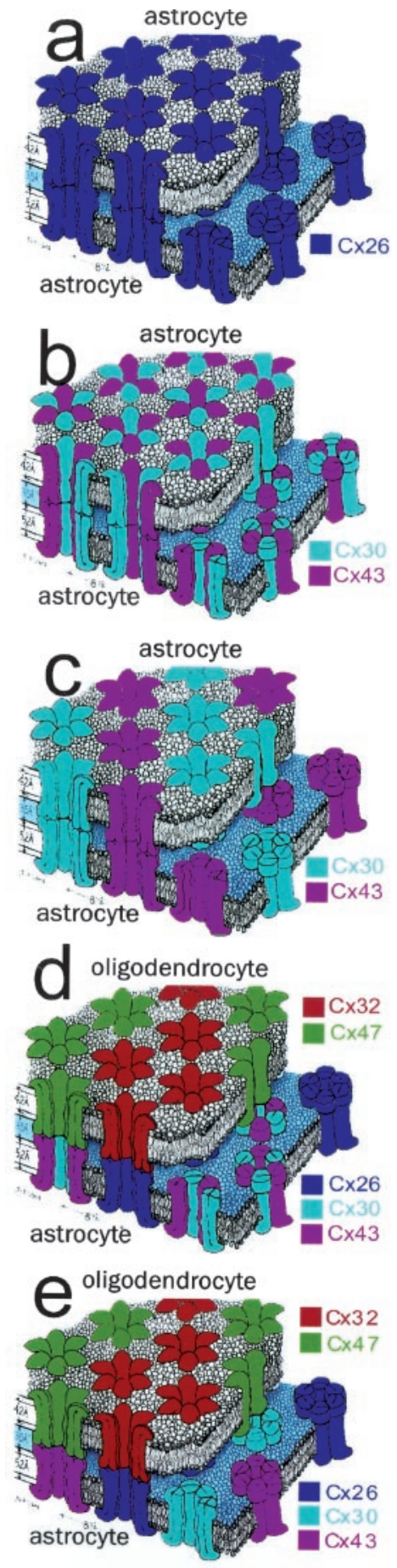

Figure 10. Multiple types of gap junctions and intercellular channels formed by astrocytes and oligodendrocytes. Astrocytes form two classes of gap junctions with each other. One class contains only $\mathrm{Cx26}(a)$, whereas a second class contain only $\mathrm{Cx} 30$ and $\mathrm{Cx} 43(b, c)$. It has not yet been determined whether $\mathrm{C} \times 30$ and $\mathrm{C} \times 43$ form heteromeric connexons. If $\mathrm{so}$, then the second class of astrocyte junction likely contains a single type of heteromeric, heterotypic intercellular channel $(b)$. If not, then they could contain several types of intercellular channels ( $c$ ). In contrast, gap junctions between astrocytes and oligodendrocytes are not separated into obligatory classes. However, all astrocyte- oligodendrocyte gap junctions contain heterotypic channels ( $d$, e). Astrocyte $C \times 26$ preferentially associates with oligodendrocyte $C \times 32$, whereas astrocyte $C \times 30$ and $\mathrm{C} \times 43$ preferentially associate with oligodendrocyte $\mathrm{C} x 47$. It remains to be determined whether $\mathrm{C} \times 47$ interacts with heteromeric connexons containing both $\mathrm{C} \times 30$ and $\mathrm{Cx} 43(d)$ or whether Cx30 and Cx43 are separated (e). Adapted from Makowski et al. (1977).
(1995) Connexin32 is a myelin-related protein in the PNS and CNS. J Neurosci 15:8281-8294.

Sontheimer H, Waxman SG, Ransom BR (1991) Relationship between $\mathrm{Na}^{+}$ current expression and cell-cell coupling in astrocytes cultured from rat hippocampus. J Neurophysiol 65:989-1002.

Stout CE, Costantin JL, Naus CC, Charles AC (2002) Intercellular calcium signaling in astrocytes via ATP release through connexin hemichannels. J Biol Chem 277:10482-10488.

Sutor B, Schmolke C, Teubner B, Schirmer C, Willecke K (2000) Myelination defects and neuronal hyperexcitability in the neocortex of connexin 32-deficient mice. Cereb Cortex 10:684-697.

Waxman SG, Black JA (1984) Freeze-fracture ultrastructure of the perinodal astrocyte and associated glial junctions. Brain Res 308:77-87.

Ye ZC, Wyeth MS, Baltan-Tekkok S, Ransom BR (2003) Functional hemichannels in astrocytes: a novel mechanism of glutamate release. J Neurosci 23:3588-3596. 